\title{
Impact of Asymmetrical Disturbance Events on Voltage Sag Source Detection
}

\author{
Boštjan Polajžer, Gorazd Štumberger, Sebastijan Seme, Drago Dolinar. \\ University of Maribor \\ Faculty of Electrical Engineering and Computer Science \\ Smetanova 17, 2000 Maribor, Slovenia \\ phone:+386 2220 7076, fax:+386 2220 7272, e-mail: bostjan.polajzer@uni-mb.si
}

\begin{abstract}
Methods for voltage sag source detection based either on energy or current criteria are discussed in this work. They are tested by applying extensive simulations and field tests. The obtained results show severe impact of asymmetrical disturbance events on voltage sag source detection, particularly in cases of upstream ground faults. However, all discussed methods show very high effectiveness in cases of voltage sags due to heavy motor starting and other symmetrical voltage sags. Based on the performed evaluation it can be concluded, that further development is still needed to increase the degree of confidence in the discussed methods for voltage sag source detection.
\end{abstract}

Key words: power systems, disturbances, source detection, voltage sag

\section{Introduction}

Among the wide range of power quality disturbances voltage sags are the most frequent ones, since they can be provoked by different events throughout the network, such as faults, motor starting, transformer energizing and heavy load switching [1]. Despite their relatively short duration-usually less than one second, voltage sags might be detrimental to several industrial loads. The detection and measurement of voltage sags is, therefore, essential for possible mitigation [2]-[3], as well as for further analysis [4]. Reliable information about a voltage sag source is indispensable in order to identify the responsible party for production losses or interruptions in the power supply. It has already been reported that it is possible to use sampled voltage and current waveforms to determine on which side of the recording device voltage sag originates, i.e. from the upstream or downstream side [5]-[7]. However, a methodology for pinpointing the exact locations of voltage sags does not as yet exist.

This paper discusses three different methods for voltage sag source detection based on instantaneous values of voltages and currents. The method proposed in [5] (method I) is based on the assumption, that the energy flow at the monitoring point increases during downstream events and decreases during upstream events. The methods proposed in [6] and [7] (methods II and III) are both based on the assumption that currents measured at the monitoring point increase during downstream events and decrease during upstream events. The slope of a current-voltage trajectory is investigated in method II, while a real current component is observed within method III.

Voltage sags are transient disturbance events, where voltages and currents are generally asymmetrical and often contain a considerable amount of aperiodic components. Thus, methods II and III, which are both phasor-based, might not be appropriate for the detection of voltage sag sources. Moreover, criteria within methods II and III are checked for each phase individually. In the cases of asymmetrical voltage sags exact interpretation of the obtained results, therefore, might not be possible. Method I is, on the contrary, based on instantaneous values, while a three-phase criterion is used. Furthermore, since most power-quality monitors can provide us only with rms values of voltages and currents and with active power, the proposed methods can be adapted to work with these signals. In this way, methods Ia, IIa and IIIa are introduced. All the discussed methods for voltage sag source detection are tested applying extensive simulations. Results obtained from the field test are also included.

\section{Methods for voltage sag source detection}

Let us consider the monitoring point shown in Fig. 1. Voltage sags might originate either from point A or from point B. In regard to energy flow direction in the steadystate, upstream and downstream events are defined in points A and B, respectively. A power-quality monitor or another recording device is placed at the monitoring point. Based on the recorded line voltages $u_{k}(t)$ and currents $i_{k}(t)$, where $k \in\{a, b, c\}$ ( $a, b$ and $c$ denote individual phases), it is possible to determine on which side of the recording device the voltage sag originated by applying the following methods.

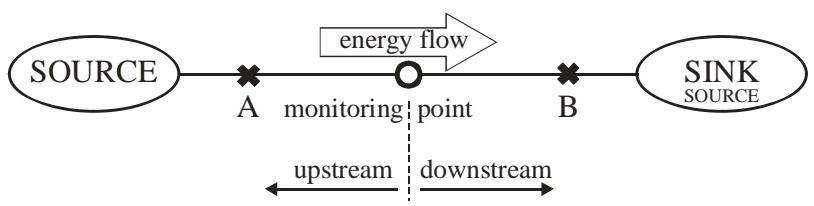

Fig. 1. Upstream event (A) and downstream event (B) 
Method I [5]: This method is based on the assumption that the energy flow at the monitoring point increases during downstream events and decreases during upstream events. The disturbance power $\Delta p(t):=p(t)-p_{\text {ss }}(t)$ is calculated, defined as the difference between the total three-phase instantaneous power $p(t)$ and the steady-state three-phase instantaneous power $p_{s s}(t)$. The disturbance energy $\Delta w(t)$ is used as the criterion (1).

$$
\Delta w(t)=\int_{0}^{t} \Delta p(\tau) d \tau\left\{\begin{array}{l}
<0 \Rightarrow \text { upstream } \\
>0 \Rightarrow \text { downstream }
\end{array}\right.
$$

Method II [6]: This method is based on the assumption that currents measured at the monitoring point increase during downstream events and decrease during upstream events, as it shown in Fig. 2. Phasors for fundamental harmonic components of line voltages $\underline{U}_{k}=\left|\underline{U}_{k}\right| \mathrm{e}^{\mathrm{j} \varphi u, k}$ and currents $\underline{I}_{k}=\left|\underline{I}_{k}\right| \mathrm{e}^{\mathrm{j} \varphi i, k}$ are calculated. $\left|\underline{U}_{k}\right|$ and $\left|\underline{I}_{k}\right|$ are phasor lengths, while $\varphi_{u, k}$ and $\varphi_{i, k}$ are phasor angles, where $k \in\{a, b, c\}$. A phase angle is defined as $\varphi_{k}:=\varphi_{u, k}-\varphi_{i, k}$. During the voltage sag, the points of $\left(\left|\underline{I}_{k}\right|,\left|\underline{U}_{k} \cos \varphi_{k}\right|\right)$ are approximated using the linear function for each phase individually, in order to investigate its slope (2) - (Fig. 2).

$$
\text { slope }\left(\left|\underline{I}_{k}\right|,\left|\underline{U}_{k} \cos \varphi_{k}\right|\right)\left\{\begin{array}{l}
>0 \Rightarrow \text { upstream } \\
<0 \Rightarrow \text { downstream }
\end{array}\right.
$$
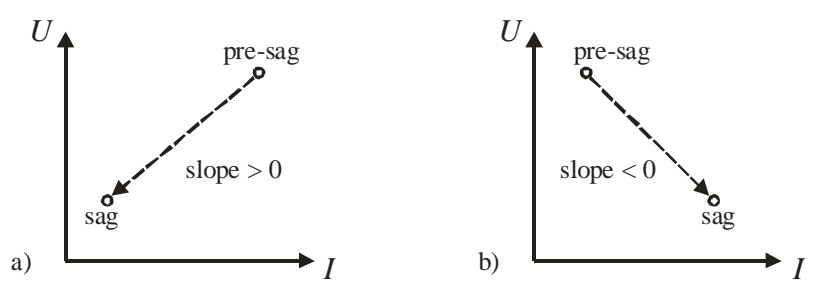

Fig. 2. $U-I$ characteristics in case of upstream event (a) and downstream event (b)

Method III [7]: This method is based on the same assumption as method II. Phasors for the fundamental harmonic components of line voltages and currents are calculated in the same way as in method II. Waveform of a real current component is calculated for a few cycles prior and during the voltage sag. The sign of its first peak at the beginning of the voltage sag is used as the criterion for each phase individually (3).

$$
\text { first peak }\left(\left|\underline{I}_{k}\right| \cos \varphi_{k}\right)(t)\left\{\begin{array}{l}
<0 \Rightarrow \text { upstream } \\
>0 \Rightarrow \text { downstream }
\end{array}\right.
$$

Since most power-quality monitors can provide us only with rms values of voltages $U_{k}(t)$ and currents $I_{k}(t)$, and with active power $P(t)$, the discussed methods I, II and III can be adapted to work with these signals. In this way, methods Ia, IIa and IIIa are introduced as follows.

Method Ia: The measured active power $P(t)$ is used instead of the instantaneous power $p(t)$, where the disturbance power $\Delta P(t)$ is introduced in the same manner as within the original method I. The disturbance energy $\Delta W(t)$ is thus used as the criterion (4).

$$
\Delta W(t)=\int_{0}^{t} \Delta P(\tau) d \tau\left\{\begin{array}{l}
<0 \Rightarrow \text { upstream } \\
>0 \Rightarrow \text { downstream }
\end{array}\right.
$$

Methods IIa and IIIa: The measured rms voltages and currents $U_{k}$ and $I_{k}$ are applied instead of the phasor lengths $\left|\underline{U}_{k}\right|$ and $\left|\underline{I}_{k}\right|$, where $k \in\{a, b, c\}$. Furthermore, instead of the phasor power factor $\cos \varphi_{k}$ the power factor defined by $\mathrm{PF}_{k}:=P_{k} /\left(U_{k} I_{k}\right)$ is used. Note that $P_{k}$ is a perphase active power, where $\sum P_{k}=P$. The method IIa is introduced by (5), where the points of $\left(I_{k}, U_{k} \cdot \mathrm{PF}_{k}\right)$ are approximated using the linear function for each phase individually in order to investigate its slope. Within method IIIa waveform of a product $\left(I_{k} \cdot \mathrm{PF}_{k}\right)$ is observed, while the sign of its first peak at the beginning of the voltage sag is used as the criterion for each phase individually (6).

$$
\begin{aligned}
& \text { slope }\left(I_{k}, U_{k} \cdot \mathrm{PF}_{k}\right)\left\{\begin{array}{l}
>0 \Rightarrow \text { upstream } \\
<0 \Rightarrow \text { downstream }
\end{array}\right. \\
& \text { first peak }\left(I_{k} \cdot \mathrm{PF}_{k}\right)\left\{\begin{array}{l}
<0 \Rightarrow \text { upstream } \\
>0 \Rightarrow \text { downstream }
\end{array}\right.
\end{aligned}
$$

\section{Results}

\section{A. Preliminary testing for asymmetrical voltage sags}

Criteria within methods II, IIa, III and IIIa are checked for each phase individually. In cases of asymmetrical voltage sags, exact interpretation of the obtained results, therefore, might not be possible. Let us consider a radial system during the upstream phase-to-phase fault, where the transformer (Dyg5) is placed between the voltage sag source and the monitoring point. Note that, according to the $A B C$ classification [8], the voltage sag observed on the transformer's primary side is, in this case, a type $C$ (only in phases $a$ and $b$ ), while that observed on the transformer's secondary side, i.e. at the monitoring point, is a type $D$ (a small sag in phases $a$ and $b$ and a large sag in phase $c$ ). The results obtained by methods II and III are show in Fig. 3, where it can be seen that they can not be exactly interpreted.
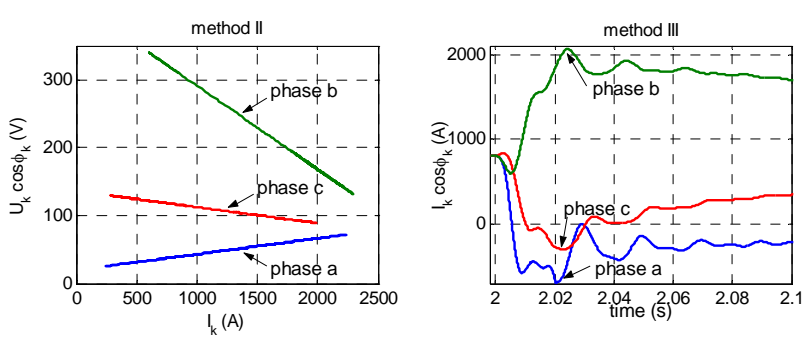

Fig. 3. Results obtained by method II and by method III for the upstream phase $a$-to-phase $b$ fault 


\section{B. Numerical simulations}

A testing-network, shown in Fig. 4, was selected for numerical simulations of voltage sags. MATLAB/Simulink-based calculations were performed. An extensive number of tests were performed for different combinations of passive and active loads, such as RL-load, induction motor-generator and synchronous motor-generator. Three types of asymmetrical faults were applied in four different locations (FL1-FL4): ground fault, phase-to-phase-to-ground fault and phase-to-phase fault. Line voltages and currents were captured at all four monitoring points (MP1-MP4). All six discussed methods for voltage sag source detection were, thus, tested for altogether 273 different examples of voltage sags due to asymmetrical disturbance events. Furthermore symmetrical voltage sags due to motor starting and three-phase faults were also simulated.

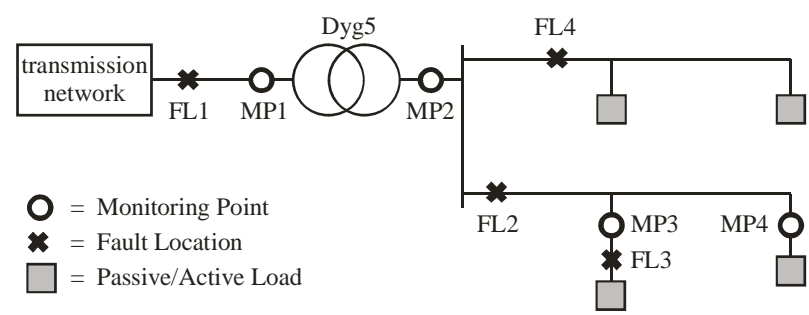

Fig. 4. Testing-network for simulations of voltage sags

A typical example of the simulation results is shown in Fig. 5 for the upstream phase-to-phase-to-ground fault in location FL2, while voltages and currents were captured at MP3 (Fig. 4). The results obtained using methods II, IIa, III and IIIa can not be interpreted exactly, since they are different for individual phases. On the contrary, methods I and Ia gave us an exact and correct results.

In Fig. 6 an example of the simulation results is shown for the downstream phase-to-phase fault in location FL3, while voltages and currents were captured on the secondary side of the power transformer (MP3 in Fig. 4). All discussed methods gave us correct results.
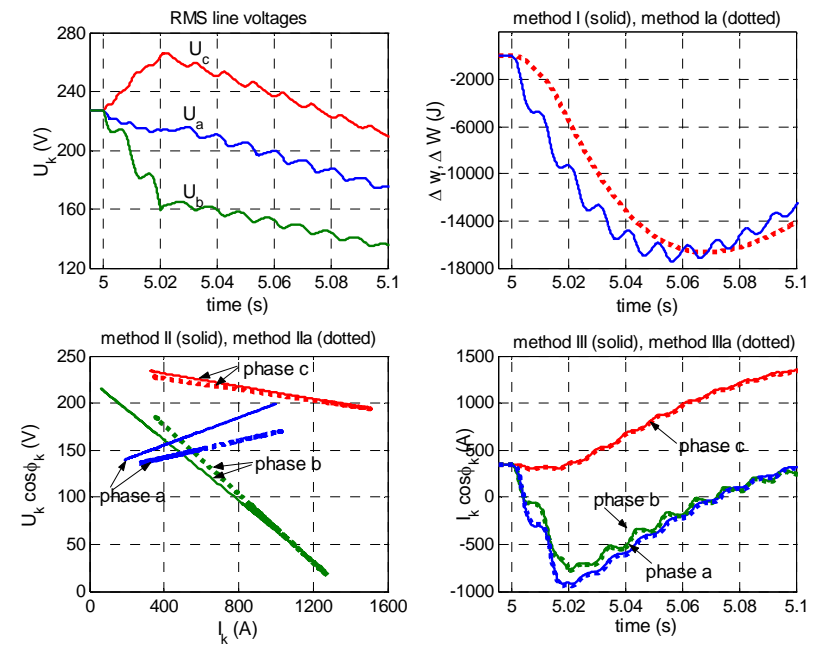

Fig. 5. Simulation results for the upstream phase-to-phase-to-ground fault.
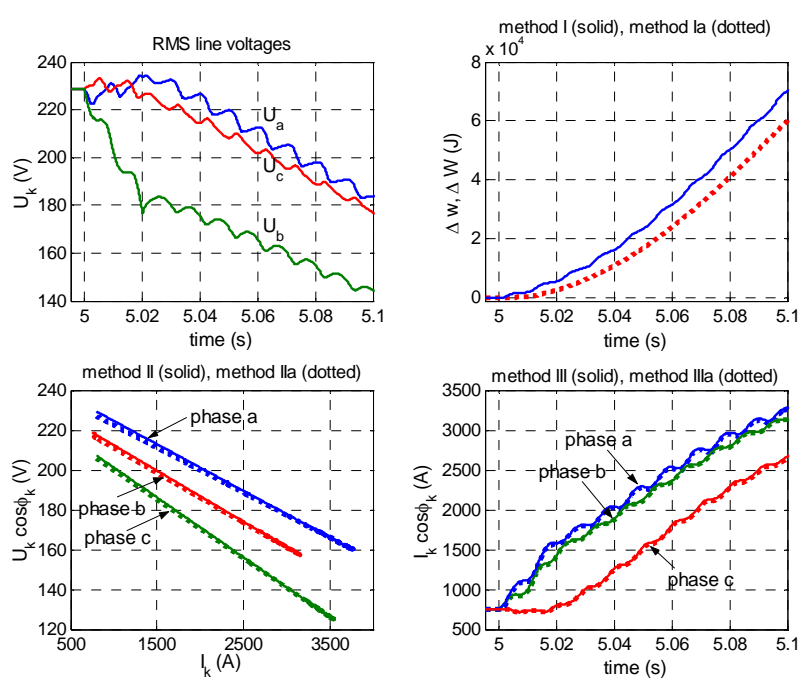

Fig. 6. Simulation results for the downstream phase-to-phase fault.

\section{Field tests}

Field tests of the discussed voltage sag source detection methods were also performed, as shown in Fig. 7. Voltages and currents were captured in a $20 \mathrm{kV}$ network and in the neighbouring $100 \mathrm{kV}$ and $0.4 \mathrm{kV}$ networks (MP1-MP3). During the field test a ground fault was generated in the $20 \mathrm{kV}$ network, provoking extremely deep voltage sag at the $20 \mathrm{kV}$ bus (MP2). This event lead to the protection-relay trip and then to the successful auto-reclosure. Subsequently, a power transformer $110 / 20 \mathrm{kV}$, connected to the $20 \mathrm{kV}$ bus, was energized. Two more voltage sags were thus provoked at the $20 \mathrm{kV}$ bus (MP2) and in the neighbouring $0.4 \mathrm{kV}$ network (MP3).

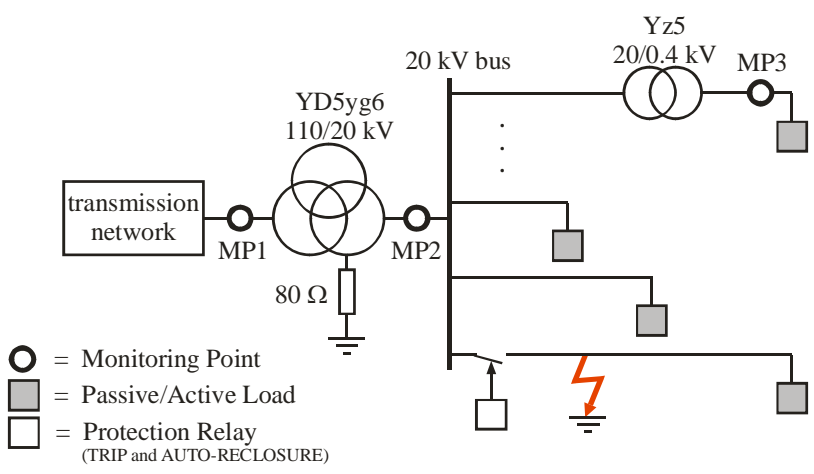

Fig. 7. Field testing

An example of field testing results is shown in Fig. 8. The discussed voltage sag was captured at the $20 \mathrm{kV}$ bus (MP2 in Fig. 7). The obtained results show typical waveforms for the rms voltages during the transformer energizing, while only methods I and Ia gave us correct results. Results obtained by methods II, IIa, III and IIIa can not be interpreted exactly, since they are different for individual phases. 

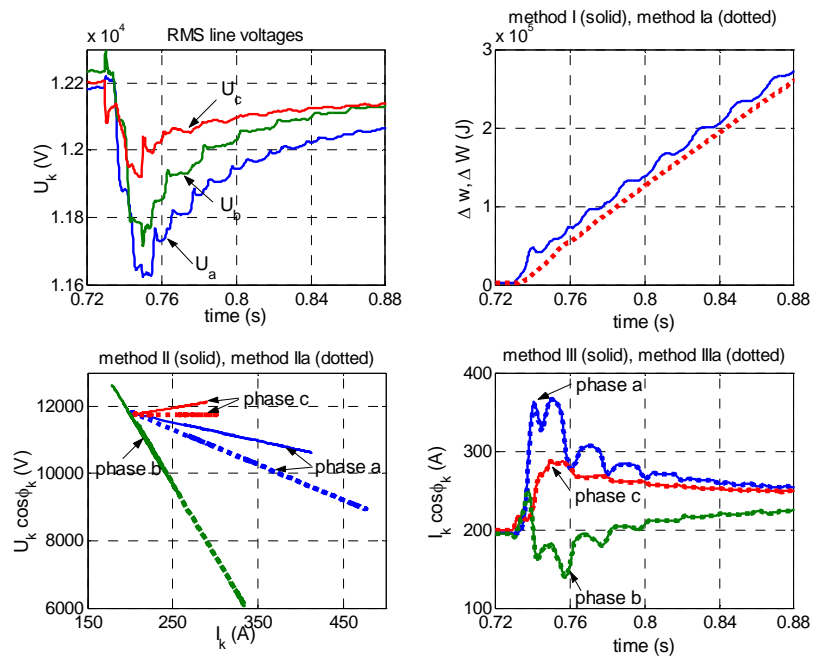

Fig. 8. Filed testing results for the downstream transformer energizing

\section{Evaluation of discussed methods for voltage sag source detection}

All the discussed methods for voltage sag source detection were tested by applying extensive numerical simulations and measurements of voltage sags. Effectiveness was determined for all six methods, where special attention was paid to voltage sags due to ground faults, since they are the most frequent ones. Effectiveness of the discussed methods was also determined for the voltage sags recorded on the secondary side of the power transformer (MP2 in Fig. 4 and 7) which connects the transmission and distribution networks.

The obtained results are summarized in Table I. It can be concluded, that method I works fine in almost all cases, except in particular cases of upstream ground faults, i.e. when the source internal impedance is relatively small. Methods II, IIa, III and IIIa, as already mentioned, do not work well in cases of asymmetrical voltage sags, especially for those originating from the upstream side. Moreover, their effectiveness is in cases of asymmetrical voltage sags even less than $50 \%$, where all 273 different examples of voltage sags were considered. The severe impact of asymmetrical disturbance events on detecting the voltage sag sources by the discussed methods is, therefore, more than evident. However, let us emphasize that all six discussed methods show very high effectiveness (more than $95 \%$ ) in cases of symmetrical voltage sags due to heavy motor starting and three-phase faults.

From the obtained results it can be seen, that method Ia shows similar properties as method I. Furthermore, method IIa shows similar properties as method II, while method IIIa shows similar properties as method III. Since most power-quality monitors can provide us only with rms values of voltages and currents and with active power, the proposed methods Ia, IIa and IIIa can be applied in such cases.
TABLE I - EFFECTIVENESS OF DISCUSSED METHODS FOR VOLTAGE SAG SOURCE DETECTION

\begin{tabular}{|c|c|c|c|c|}
\hline Method & $\begin{array}{c}\text { asymm. } \\
\text { sags (total) }\end{array}$ & $\begin{array}{l}\text { ground } \\
\text { faults }\end{array}$ & MP2 & $\begin{array}{c}\text { upstream } \\
\text { events }\end{array}$ \\
\hline II & - & ?? & •• & • \\
\hline Ia & $\bullet$ & ?? & $\bullet \bullet$ & $\bullet$ \\
\hline II & - & - & ?? & ?? \\
\hline IIa & - & - & ?? & ?? \\
\hline III & - & - & $\bullet$ & ?? \\
\hline IIIa & - & - & • & ?? \\
\hline \multicolumn{5}{|c|}{ 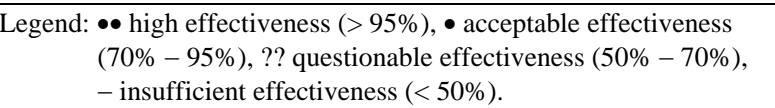 } \\
\hline
\end{tabular}

\section{Conclusion}

The impact of asymmetrical disturbance events on voltage sag source detection is studied in this paper. For this purpose, different methods for voltage sag source detection are tested by applying extensive simulations and field tests. The obtained results show that methods II and III, as well as methods IIa and IIIa do not work well, particularly in cases of asymmetrical voltage sags due to upstream events. Methods I and Ia work fine in almost all cases, except in particular cases of upstream ground faults. Based on the obtained results it can be concluded, that further development is still needed to increase the degree of confidence in the discussed methods for voltage sags source detection.

\section{Acknowledgement}

This work was supported by ARRS, Projects no. P2-0115 and L2-7560-1792.

\section{References}

[1] M. J. H. Bollen, "Voltage sags in three-phase systems," IEEE Power Engineering Review, vol. 21, no. 9, pp. 8-11,15, September 2001.

[2] M. Karimi, H. Mokhtari and M. R. Iravani, "Wavelet based on-line disturbance detection for power quality applications," IEEE Transactions on Power Delivery, vol. 15, no. 4, pp. 1212-1220, October 2000.

[3] C. Fitzer, M. Barnes and P. Green, "Voltage sag detection technique for a dynamic voltage restorer," IEEE Transactions on Industry Applications, vol. 40, no. 1, pp. 203-212, January 2004.

[4] J. Arrillaga, M. J. H. Bollen and N. R. Watson, "Power quality following deregulation," Proceedings of the IEEE, vol. 88, no. 2, pp. 246-261, February 2000.

[5] A. C. Parsons, W. M. Grady, E. J. Powers and J. C. Soward, "A direction finder for power quality disturbances based upon disturbance power and energy," IEEE Transactions on Power Delivery, vol. 15, no. 3, pp. 1081-1086, July 2000.

[6] C. Li, T. Tayjasanant, W. Xu and X. Liu, "Method for voltagesag-source detection by investigating slope of the system trajectory," IEE Proceedings: Generation, Transmission and Distribution, vol. 150, no. 3, pp. 367-372, May 2003.

[7] N. Hamzah, A. Mohamed and A. Hussain, "A new approach to locate the voltage sag source using real current component," Electric Power Systems Research, vol. 72, no. 2, pp. 113-123, December 2004.

[8] M. J. H. Bollen, Understanding power quality problems: voltage sags and interruptions. IEEE Press, New York, 2000. 